\title{
Light-driven biological actuators to probe the rheology of 3D microtissues
}

Adrien Méry, Artur Ruppel, Jean Révilloud, Martial Balland, Giovanni Cappello and Thomas Boudou*

\begin{abstract}
Affiliations:
Laboratory of Interdisciplinary Physics (LIPhy), University Grenoble Alpes, CNRS, F-38000 Grenoble, France
\end{abstract}

\section{* Corresponding author}

Email: thomas.boudou@univ-grenoble-alpes.fr;

\section{Abstract}

The mechanical properties of biological tissues are key to the regulation of their physical integrity and function. Although the application of external loading or biochemical treatments allows to estimate these properties globally, it remains problematic to assess how such external stimuli compare with internal, cell-generated contractions. Here we engineered 3D microtissues composed of optogenetically-modified fibroblasts encapsulated within collagen. Using light to control the activity of RhoA, a major regulator of cellular contractility, we induced local mechanical perturbation within 3D fibrous microtissues, while tracking in real time microtissue stress and strain. We thus investigated the dynamic regulation of light-induced, local contractions and their spatio-temporal propagation in microtissues. By comparing the evolution of stresses and strains upon stimulation, we demonstrated the potential of our technique for quantifying tissue elasticity and viscosity, before examining the possibility of using light to map local anisotropies in mechanically heterogeneous microtissues. Altogether, our results open an avenue to non-destructively chart the rheology of 3D tissues in real time, using their own constituting cells as internal actuators. 


\section{Introduction}

Tissue engineering holds great potential to develop organotypic in vitro model systems [Ref review]. With their miniaturization in the late 2000's ${ }^{1,2}$, such three-dimensional (3D) microtissue models have been used for studying fundamental features of tissue biology such as the repair of wounded fibrous tissue ${ }^{3}$, or the formation and maturation of myocardial ${ }^{4}$ and lung tissues ${ }^{5}$. Their sub-millimetric size has also opened the possibility to use high-throughput, low volume screening of drugs ${ }^{6,7}$ or functional effects of patient-specific mutations ${ }^{8}$.

By combining microtissue engineering with magnetic or vacuum actuation, the tissue mechanical properties have recently been assessed and their importance in the tissue formation and physiological function evidenced $^{9,10}$. For example, Zhao et al. demonstrated the respective roles of cell-generated forces and collagen structures in tissue mechanics ${ }^{9,11,12}$ while Walker et al. connected remodeling of the cytoskeleton to homeostatic mechanical regulation of tissues ${ }^{10,13}$. A key limitation to the study of mechanical signals in tissue biology is the difficulty to control, over space and time, the tensional state of cells. Indeed, while both magnetic or vacuum actuations allow for applying an external stretch to the tissue, it remains problematic to assess how such external mechanical stimulations compare with internal, cell-generated contractions. Indeed, no method currently exists that can locally modulate the contractility of a group of cells within a 3D tissue while simultaneously measuring global tissue contractility as well as both fine-scale cytoskeletal and extracellular architecture.

Thanks to its spatial and temporal resolution, optogenetics has emerged as a powerful tool for spatiotemporally controlling cell signaling ${ }^{14}$. Using optogenetic control of RhoA activity, several studies recently used light pulses to locally up- or down-regulate cell-generated forces ${ }^{15,16}$. Valon et al. further demonstrated that these changes in cellular tension were paralleled by tissue deformations in 2D epithelial monolayers ${ }^{16}$, suggesting an exciting avenue for using cells as mechanical actuators. Such biological actuators could be employed to probe tissue mechanics using light-induced physiological mechanical stimuli. 
We combine here 3D microtissues and optogenetics, by engineering 3D microscale constructs of optogenetically-modified fibroblasts embedded within collagen 3D matrices. Using light to control the proximity of RhoA and one of its activator ARHGEF11, we modulate cellular contractility within 3D fibrous microtissues, while microcantilevers report microtissue stress in real time. We demonstrate our ability to control and measure, over space and time, the stress of specific parts of microtissues while simultaneously inferring tissue strain using particle image velocimetry (PIV). We thus investigate the elasticity and viscosity of microtissues, before demonstrating the potential of our technique for quantifying the impact of the mechanical stiffness of the cantilevers and the extracellular matrix on the tissue rheology. We then demonstrate the ability of our approach to map local anisotropies in mechanically heterogeneous microtissues. Together, these results highlight a unique approach to examine the effects of various parameters such as mechanical preload, tissue maturation, matrix architecture and stiffness on both the ability to propagate physiological mechanical signals and the dynamic mechanical properties of engineered fibrous microtissues.

\section{Results}

\section{Optogenetic stimulation of microtissues}

We used optogenetics to generate contractions in parts of engineered microtissues (Fig. 1.A). Our strategy was to trigger the activation of the small GTPase RhoA, a major regulator of cellular contraction ${ }^{17}$. To this end, we used NIH3T3 cells stably expressing a Cry2-CIBN optogenetic probe (opto-RhoA fibroblasts) to dynamically control with blue light the localization of ArhGEF11, an upstream regulator of RhoA ${ }^{18}$. As previously described, ArhGEF11 was recruited to the cell membrane upon blue light stimulation, thus activating RhoA and subsequently cell contractility (Fig. 1.B) ${ }^{16}$. We embedded these opto-RhoA fibroblasts in a neutralized collagen I solution within microfabricated PDMS wells containing two T-shaped microcantilevers. Over time of cultivation, the fibroblasts spread inside the collagen and spontaneously compacted the matrix to form opto-RhoA microtissues that 
spanned across the top of the pair of cantilevers, which deflection was measured to quantify tissue tension ${ }^{2}$. During tissue formation, a baseline static tension developed due to compaction of the gel by the collective action of the fibroblasts. Once the tissue formed, we used a digital micro-mirror device (DMD) to illuminate parts of these opto-RhoA microtissues. To demonstrate our ability to apply local stimulations, we consecutively illuminated the left and right halves of microtissues while measuring the microtissue tension as well as the local displacements using PIV (Fig. 1.C, supp. movie 1). Immediately after a pulse of light (characterized by an irradiance of $1.7 \mathrm{~mW} / \mathrm{mm}^{2}$ and a duration of $500 \mathrm{~ms}$, i.e. an energy of $0.9 \mathrm{~mJ} / \mathrm{mm}^{2}$ ), the tissue in the stimulated area contracted, inducing a quick increase in tissue tension (half-time to maximum increase $t_{\frac{1}{2}}^{i}=26 \pm 8 \mathrm{~s}$ ), before slowly relaxing back to baseline (half-time of decrease from maximum $t_{\frac{1}{2}}^{d}=317 \pm 54$ s) (Fig. 1.C-D). Fig. 1.C shows the corresponding local contractions of the left and right halves of the microtissues. For both stimulations patterns, the microtissue tension increased to a similar level and decreased back to baseline level, thus indicating an elastic response without plastic deformation. Moreover, the local displacements were mostly directed along the longitudinal axis, suggesting a strongly anisotropic contraction. To further investigate the geometry of deformation, we next stimulated isotropic, discoidal area of microtissues suspended between two and four micropillars. 


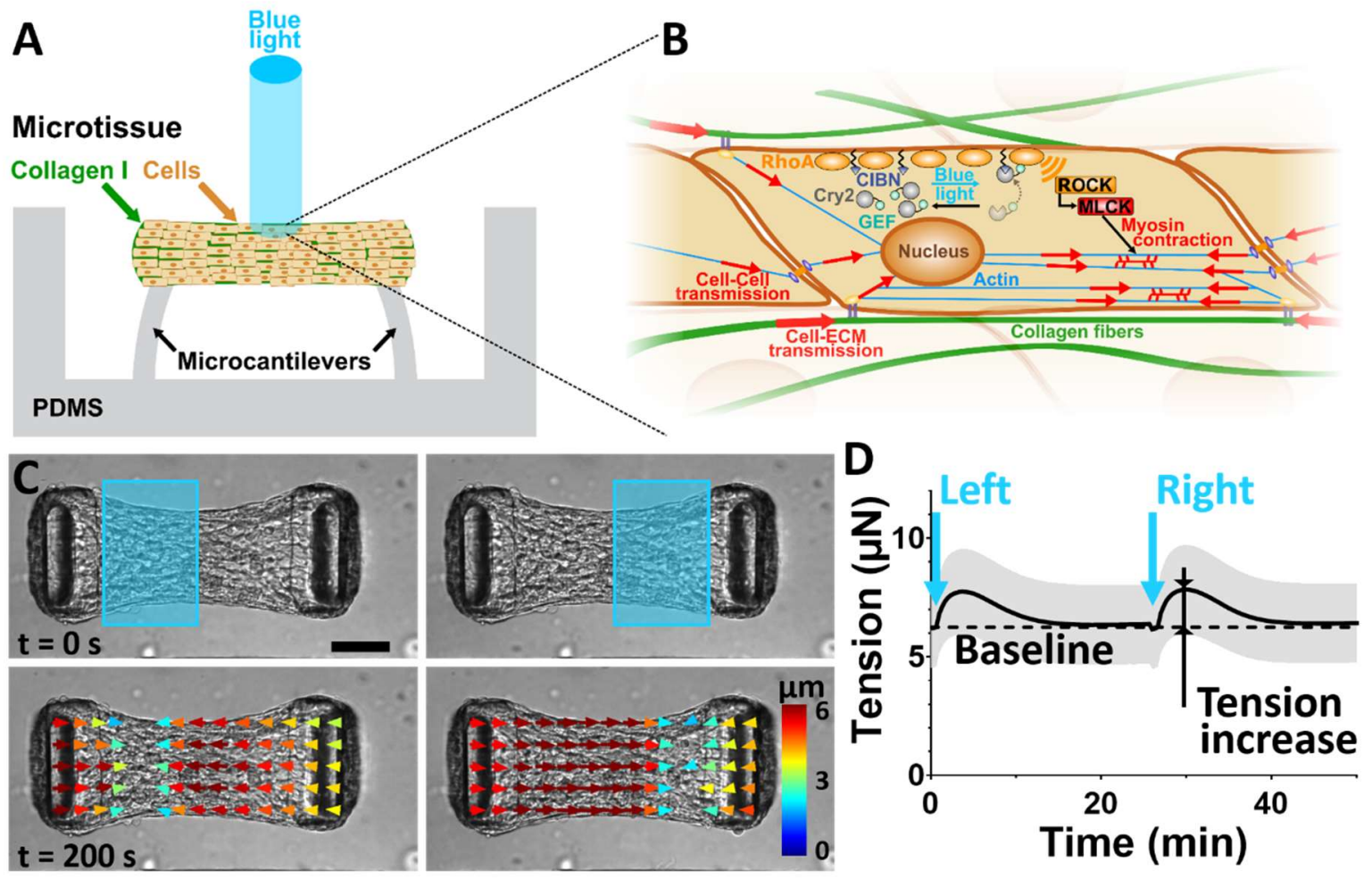

Figure 1. Light-controlled contraction of microtissues. (A) Schematic illustrating a microtissue composed of Opto-RhoA fibroblasts embedded in collagen and suspended between two flexible micropillars, whose bending reports tissue tension. (B) Schematic of the ARHGEF11(DHPH)-CRY2/CIBN-CAAX optogenetic system to control cellular contractility. Upon blue light stimulation, CRY2 changes conformation and binds to CIBN, bringing the RhoA-activator ARHGEF11 in close proximity of RhoA, which in turn induces a contractility increase. (C) Upon blue light illumination of its left- or right-half, the microtissue contracts locally, as shown by the PIV-tracking of the displacements. For readability reasons, only half of the vectors are represented. Scale bar is $100 \mu \mathrm{m}$. (D) Temporal evolution of the tissue tension upon the simulation of its left- and right-half. Data are the average of 25 microtissues \pm SD.

The optogenetically-induced contraction follows the anisotropy of the microtissue

\section{architecture}

A major advantage of optogenetics over drug or genetic approaches is the possibility to stimulate spatially defined areas of a tissue of interest. We thus investigated the mechanical response of optomicrotissues of which only a small area is stimulated with blue-light. We stimulated a $50 \mu \mathrm{m}$ diameter disc in the center of the microtissue (Fig. 2.A, supp. movie 2) and mapped the resulting displacements over the whole microtissue by using particle image velocimetry (PIV). We observed that the stimulated central region barely moved upon illumination while the left and right parts of the microtissues were displaced inwards, toward each other, indicating compaction of the stimulated area (Fig. 2.B). Despite 
an isotropic light stimulation, the resulting displacements were strongly anisotropic, with a mean angle of $16 \pm 7^{\circ}$ and more than $80 \%$ of the displacements presenting a lower than $30^{\circ}$ angle with the longitudinal $\mathrm{x}$-axis of the tissue (Fig. 2.C). This anisotropic contraction was paralleled with a strongly anisotropic field of deformation, inferred from the displacement field, as the $x$-component of the strain $\left(\varepsilon_{x x}\right)$ was more than 15 -times larger than its y-component ( $\left.\varepsilon_{y y}\right)$ (Fig. 2.D-F, supp. movie 2). We defined the anisotropy coefficient (AC) as the difference between $\varepsilon_{x x}$ and $\varepsilon_{y y}$, normalized by their sum. In the case of these microtissues suspended between two micropillars, we measured an AC of $1.2 \pm 0.2$, indicating a strong anisotropy along the $\mathrm{x}$-axis. This quasi-1D response suggests a strong anisotropy of either the cytoskeleton of the constituting cells or the surrounding matrix. We simultaneously stained actin and collagen in microtissues and quantified their alignment. We thus found that the longitudinal contraction induced by light correlated with a strongly anisotropic architecture of both actin and collagen, with more than $80 \%$ of the actin fibers and more than $70 \%$ of the collagen fibers aligned within less than $30^{\circ}$ of the $x$-axis (Fig. 2.G-L, sup. movie 3). These data are coherent with the fact that fibroblasts line up and remodel their extracellular matrix to align with the principal maximum strains developed during tissue formation ${ }^{19-21}$. Furthermore, we show here using optogenetics that any additional contraction, even local, is constrained by the anisotropic conformation of the tissue. 

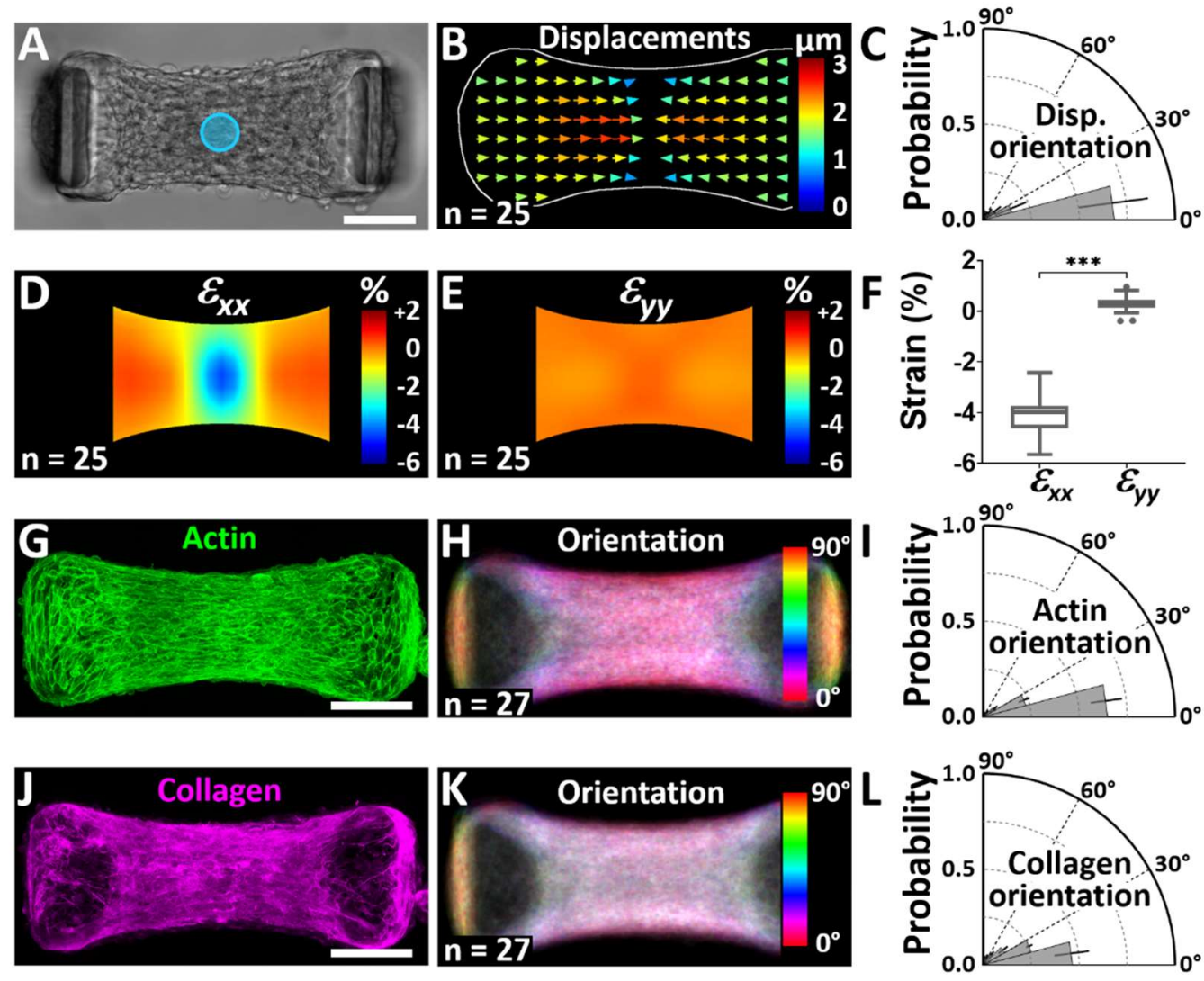

Figure 2. The anisotropy of the optogenetically-induced contraction correlates with the microtissue architecture (A) Representative microtissue where the blue disc represents the $50 \mu \mathrm{m}$ diameter isotropic stimulation area. (B) Resulting average displacement field (for readability reasons, only half of the vectors are represented) and (C) polar plot of its orientation. Data are presented as mean \pm SD with $n=25$ microtissues. Resulting average strain fields $\left(\varepsilon_{x x}\right.$ in $\mathrm{D}, \varepsilon_{y y}$ in $\mathrm{E}$ ) and comparison of the average strain amplitudes in the area of stimulation (F). Data are presented as Tukey box plots with $\mathrm{n}=25$ microtissues. ${ }^{* *} \mathrm{p}<0.001$ between $\varepsilon_{x x}$ and $\varepsilon_{y y}(\mathrm{G})$ Confocal projection of the fluorescent staining of actin in a representative microtissue. (H) Average color-coded map and (I) polar plot showing the anisotropic orientation of actin fibers along the x-axis. $(\mathrm{J})$ Confocal projection of the fluorescent staining of collagen in a representative microtissue. (K) Average color-coded map and ( $\mathrm{L}$ ) polar plot showing the anisotropic orientation of collagen fibers along the $x$-axis. Data of actin and collagen orientations are presented as mean \pm SD with $\mathrm{n}=27$ microtissues. Scale bars are $100 \mu \mathrm{m}$.

\section{Dynamics of optogenetically-induced strains}

Having shown the quasi-1D deformation of anisotropic microtissues spanning between two micropillars, we next sought to further investigate the spatio-temporal strain patterns induced by such local, optogenetically-induced contractions. We stimulated the left half of microtissues and used PIV to map the resulting displacements and infer the corresponding strains (Fig. 3, supp. movie 4). We observed that the stimulated half left of the microtissue was compressed, up to $\varepsilon_{C}^{\max }=-3.0 \pm 0.5 \%$, while the non-stimulated half right of the tissue was stretched, up to $\varepsilon_{S}^{\max }=1.0 \pm 0.3 \%$. This result indicates that when stimulated cells contract, compact and compress their surroundings. This 
compression is equilibrated by both the stretch of the non-stimulated part of the tissue and the deflection of the micropilllars. Figure 3. C-D shows the evolution of tissue stress (i.e. the tissue tension obtained from the pillar deflection divided by the cross-sectional areas of the tissue), compression and stretch over time, indicating two time delays $\tau_{c}=70 \pm 39 \mathrm{~s}$ between the maximum stress and the maximum compression, and $\tau_{s}=200 \pm 64 \mathrm{~s}$ between the maximum stress and the maximum stretch.

By stimulating only one part of the microtissue, we were thus able to simultaneously measure both the stress $\sigma_{x x}$, deduced from the pillar deflection and the cross-section of the microtissue, and the strain $\varepsilon_{x x}$, inferred from the PIV tracking of local displacements (Fig. 3. E). These results suggest the possibility to use optogenetic stimulation for assessing the mechanical properties of the microtissue.
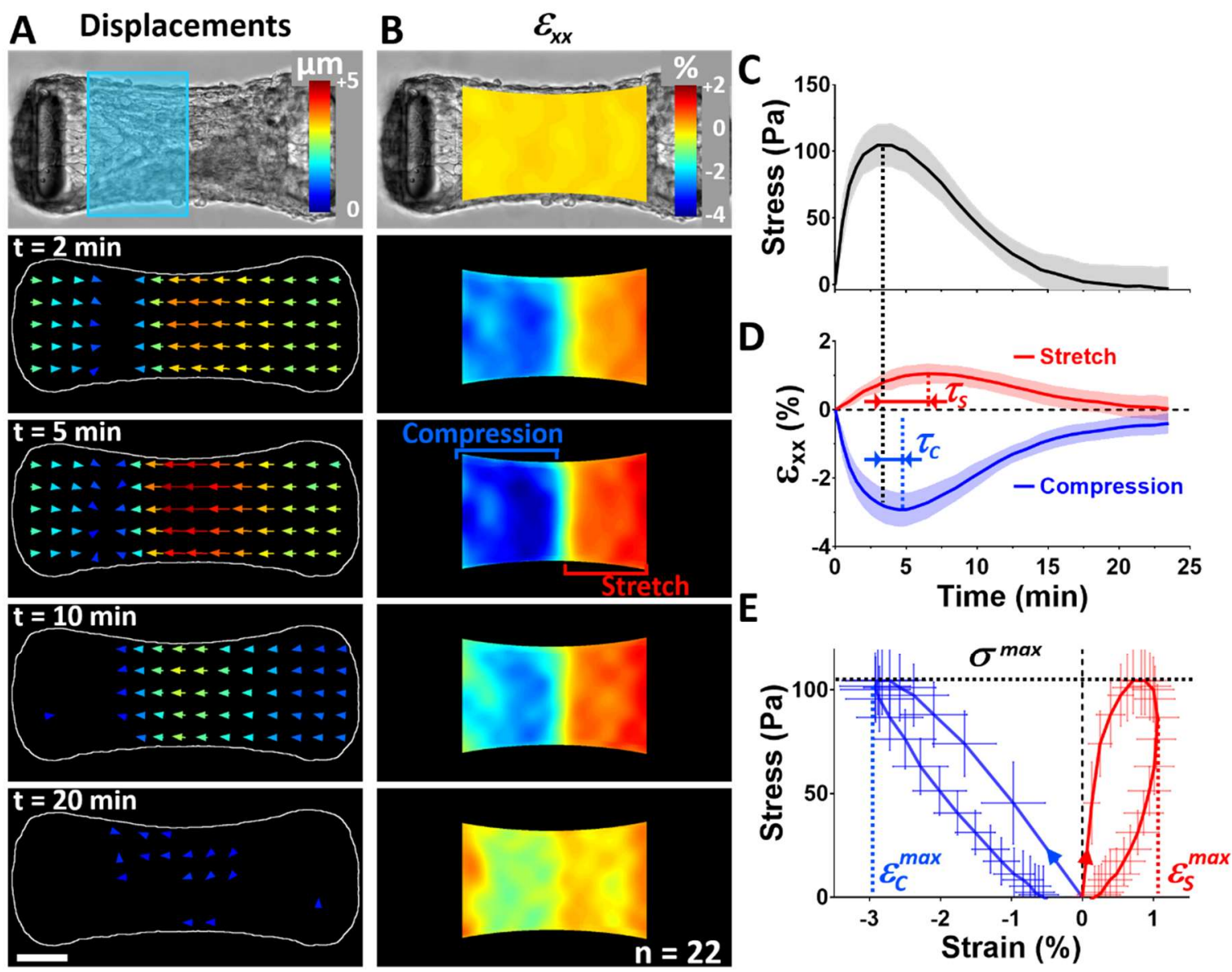

Figure 3. Light-induced local contractions evidence the viscoelastic properties of microtissues. Temporal evolution of the average displacement $(A)$ and $\varepsilon_{x x}$ strain field $(B)$ after stimulation of the left half of the tissue by blue light (symbolized by a blue rectangle in A). (C) Evolution of the stress $\sigma_{x x}$ over time and (D) resulting strain $\varepsilon_{x x}$ decomposed in its positive (stretch, in red) and negative (compression, in blue) components. (E) Corresponding stress-stretch (in red) and stress-compression (in blue) curves. The dotted lines indicate the maximum stress $\sigma^{\text {max }}$ (in black), the maximum stretch $\varepsilon_{S}^{\max }$ (in red) and the maximum compression $\varepsilon_{C}^{\max }$ (in blue). Data are the average of 22 microtissues \pm SD. 


\section{Inferring mechanical properties of stretched areas}

In order to infer the rheology of the microtissue, we assimilated the stimulated part of the tissue to an actuator stretching the non-stimulated part. We first compared the amplitudes of stress and stretch while varying the width of the stimulated area from 20 to $100 \mu \mathrm{m}$ (Fig. 4, supp. movie 5). We thus demonstrated that the force increase was proportional to the area of the stimulated zones (Fig. 4.A and Sup. Fig. 1.A-B). As the cell density is roughly homogenous along the whole length of the microtissue (Sup. Fig. 1.C-H), this result indicates that the light-induced contraction is directly proportional to the number of stimulated cells. As the contraction is quasi-1D, the cross-section of the microtissue is unchanged when the width of stimulation is varied, and the resulting, optogeneticallyinduced stress increase is also proportional to the width of stimulation, ranging from $98.5 \pm 11.2 \mathrm{~Pa}$ to $223.9 \pm 32.7 \mathrm{~Pa}$ when the stimulation width increases from 20 to $100 \mu \mathrm{m}$, respectively (Fig. 4.C).

As a result, the average stretch of the non-stimulated part increased from $0.6 \pm 0.2 \%$ to $1.2 \pm 0.3 \%$ for the same range of stimulation width (Fig. 4.B-C, supp. movie 5). Upon actuation by the stimulated part, the non-stimulated part of the tissue thus exhibits viscoelastic characteristics when undergoing deformation. The maximum stretch $\varepsilon_{S}^{\max }$ is linearly depending on the maximum applied stress $\sigma_{\max }$ although peaking with a delay $\tau_{s}$, and both stress and strain returns to 0 after stimulation. Consequently, we defined the apparent elastic modulus of the tissue $E$ as the maximum stress $\sigma^{\max }$ divided by the maximum stretch $\varepsilon_{S}^{\max }$ and observed that $E$ was logically independent of the stimulation width, with an average value of $19.2 \pm 5.0 \mathrm{kPa}$ (Fig. 4.D). Although we used different parameters, this value is in very good agreement with previous measurements we and others obtained by stretching microtissues using magnetically actuated microcantilevers (Fig. Supp. 2) ) $^{9,11}$. Similarly, we did not measure any significant difference in the time delay $\tau_{s}$ between maximum stress and stretch (Fig. 4.D), i.e. the measured viscosity of the microtissue is independent of the size of the stretched part, thus demonstrating that this viscosity is not a poroelastic effect occurring through the 
redistribution of the fluid within the microtissue but rather to the relaxation of the actomyosin cytoskeleton.
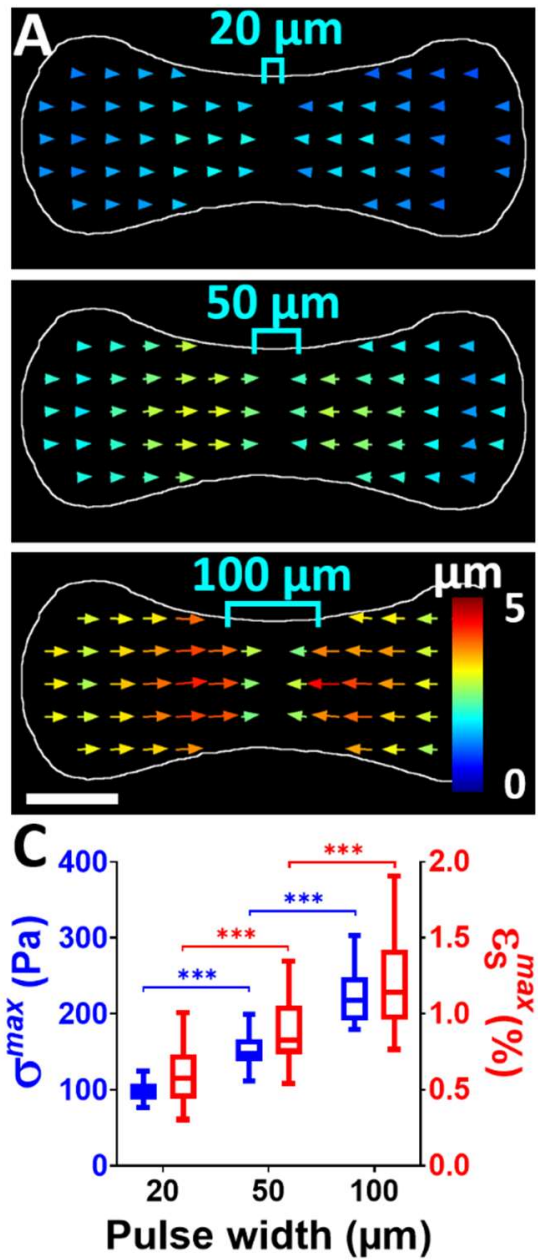
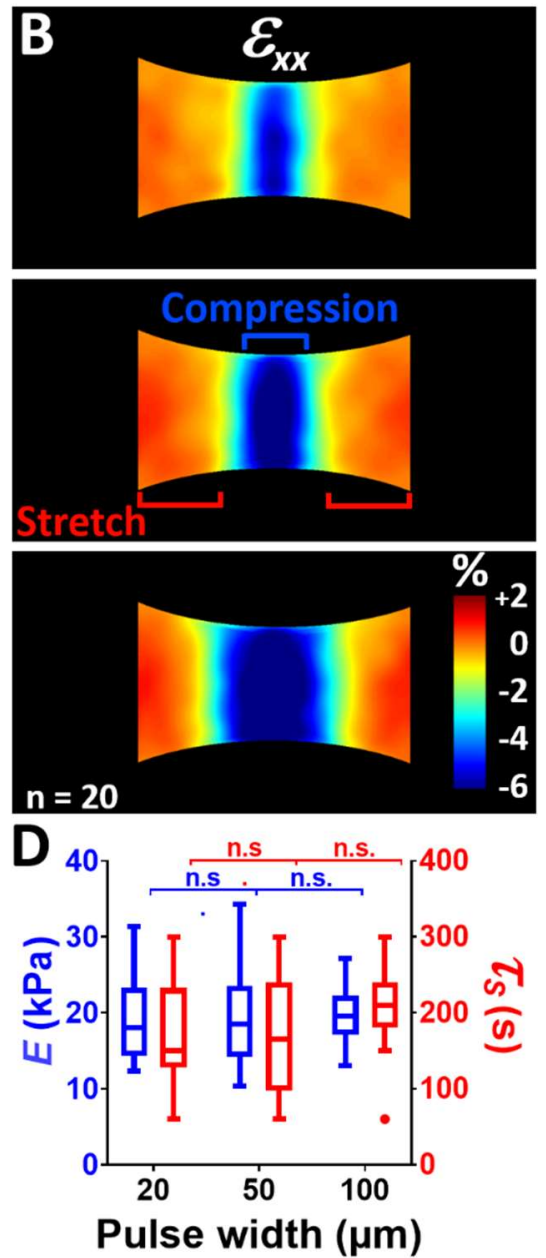

Figure 4. Light-driven probing of the apparent elastic modulus of microtissues. Average displacement ( $A$ ) and strain (B) fields resulting from $20 \mu \mathrm{m}, 50 \mu \mathrm{m}$ and $100 \mu \mathrm{m}$ wide light stimulations (symbolized by blue brackets). For readability reasons, only half of the displacement vectors are represented. Scale bar is $100 \mu \mathrm{m}$. (C) Corresponding maximum stress $\sigma^{\max }$ and stretch $\varepsilon_{S}^{\max }$. (D) Resulting elastic modulus $E$, reported as $\sigma^{\max } / \varepsilon_{S}^{\max }$, and time delay $\tau_{S}$ between reported as $\sigma^{\max }$ and $\varepsilon_{S}^{\max }$ in function of the pulse width. Data are presented as Tukey box plots with $\mathrm{n}=20$ microtissues. $* * * p<0.001$ between conditions. n.s. stands for non-significant (i.e. $p>0.05$ ).

We then tested the robustness of our approach by assessing the rheological properties of microtissues under different conditions (Fig. Supp. 2). We first investigated the influence of the boundary conditions on the rheological properties of the microtissue. To this end, we stimulated locally microtissues suspended between micropillars presenting a spring constant between 0.20 to $1.12 \mathrm{~N} / \mathrm{m}$. We measured the resulting stretch of the non-stimulated zones and inferred elastic moduli and temporal 
delay between maximum stress and stretch. In good agreement with previous measurements using various spring constants ${ }^{9}$, we found that the resting stress as well as the elastic modulus increase from $6.2 \pm 2.5$ to $21.4 \pm 8.3$ with increasing micropillar spring constant (Fig. Supp. 2). We also observed, for the first time to our knowledge, that the microtissue viscosity strongly increased with the rigidity of the boundaries, as the delay $\tau_{s}$ between maximum stress and stretch more than doubled, from $133 \pm$ $54 \mathrm{~s}$ to $282 \pm 75 \mathrm{~s}$, when the micropillar spring constant was increased from 0.20 to $1.12 \mathrm{~N} / \mathrm{m}$. As cellular contractility is the main regulator of tissue tension, while collagen architecture predominantly impacts the tissue stiffness ${ }^{9,11}$, our results suggest that the viscoelastic behavior of microtissues is due to the rupturing and reforming of bonds within the cytoskeleton (i.e. between actin filaments or between actin and myosin) ${ }^{13}$, in coherence with the previously shown stretch-induced perturbation of the acto-myosin cytoskeleton ${ }^{22,23}$.

We then probed the mechanical properties of microtissues $24 \mathrm{~h}$ and $48 \mathrm{~h}$ after seeding. We observed an almost doubling of the elastic modulus from $15.1 \pm 6.5 \mathrm{kPa}$ to $28.5 \pm 11.1 \mathrm{kPa}$ over these $24 \mathrm{~h}$, while the change in viscosity was not significant (Fig. Supp. 2). Since collagen was shown to be a key component of the tissue stiffness ${ }^{9}$, we also assessed the rheological properties of microtissue composed of different collagen densities. We measured a very slight increase of stiffness from $15.1 \pm$ 6.5 to $19.0 \pm 7.0 \mathrm{kPa}$ for initial collagen densities varying between 1.5 and $2.5 \mathrm{mg} / \mathrm{mL}$, once again without any significant change of viscosity (Fig. Supp. 2). These values correlate with previous measurements ${ }^{9}$ and the small variation of stiffness despite the large difference in initial collagen density is coherent with the insensitivity of stiffness to density for collagen under tension ${ }^{24}$ However, since collagen density feedbacks over time to regulate cell forces and stiffness, we made good use of our non-destructive approach to assess the possibly rapid changes of tissue stiffness and viscosity upon collagenase treatment. After $10 \mathrm{~min}$ of culture with $50 \mu \mathrm{g} / \mathrm{mL}$ of collagenase type I, we measured a strong decrease in tissue stiffness from $21.4 \pm 8.3$ to $9.1 \pm 6.0 \mathrm{kPa}$. However, the time delay between maximum stress and stretch was exactly the same before and after collagenase treatment. 
These results thus demonstrate that the cleavage of the peptide bonds of collagen by the collagenase strongly impacted the collagen structural integrity without affecting the microtissue viscosity.

Altogether, these results confirm the major role of collagen structure in the global stiffness of microtissues, whereas microtissue viscosity is mostly regulated by the contractile prestress of cells. As the collagen structure depends on the microtissue geometry ${ }^{21}$, we next sought to probe local mechanical anisotropies using our optogenetic approach.

\section{Optogenetic assessment of local anisotropies}

As microtissues suspended between two micropillars exhibit a pronounced, homogeneous anisotropy along the x-axis (Fig. 2), we generated microtissues suspended between four micropillars, in order to induce a more heterogeneous architecture presenting various degrees of anisotropy ${ }^{21}$. We then performed a discoidal, isotropic light stimulation either in the center or on the left side of these square microtissues (Fig. 5.A-B, supp. movie 6-7). We thus observed a completely isotropic displacement field for the centered stimulation, with a mean angle of $44 \pm 4^{\circ}$, whereas the eccentric stimulation induced a slightly biased displacement field with a mean angle of $53 \pm 4^{\circ}$ and more than $45 \%$ of the displacements oriented within less than $30^{\circ}$ of the y-axis (Fig. 5. C-E, supp. movie 6-7). These fields of displacement were paralleled with either an isotropic strain field for the centered stimulation, i.e. $\varepsilon_{x x}$ $=\varepsilon_{y y}=-1.6 \pm 0.2 \%$, or an anisotropic strain field for the eccentric stimulation with $\varepsilon_{x x}=-0.9 \pm 0.2 \%$ while $\varepsilon_{y y}=-1.6 \pm 0.3 \%$ (Fig. 2.F-J, supp. movie 6-7). We thus obtained an A.C. of $0.0 \pm 0.1$ for the center part of the tissue whereas its left side exhibited an A.C. of $-0.3 \pm 0.1$, indicating a moderate anisotropy along the $y$-axis Fig. (5.K), similarly to previous results obtained utilizing finite element models ${ }^{21}$. We then sought to compare these local anisotropic properties to the structural organization of these square microtissues. We simultaneously stained actin and collagen and quantified their respective orientations (Fig. 5.L-Q, supp. movie 8). In good agreement with previous studies ${ }^{21,25}$, we found a mostly random organization of actin and collagen fibers in the center of the microtissues, while $65 \%$ of the actin fibers and $56 \%$ of the collagen aligned within $30^{\circ}$ of the $y$-axis. This correlation between 
mechanical anisotropy, probed via optogenetics, and fine-scale cytoskeletal and extracellular architecture demonstrate that the spatial propagation of mechanical signals in microtissues is dependent upon actin and collagen organization. Consequently, the use of optogenetically-modified cells as actuators to probe their environment appears as a powerful tool to probe non-destructively architectural heterogeneities in complex tissues.
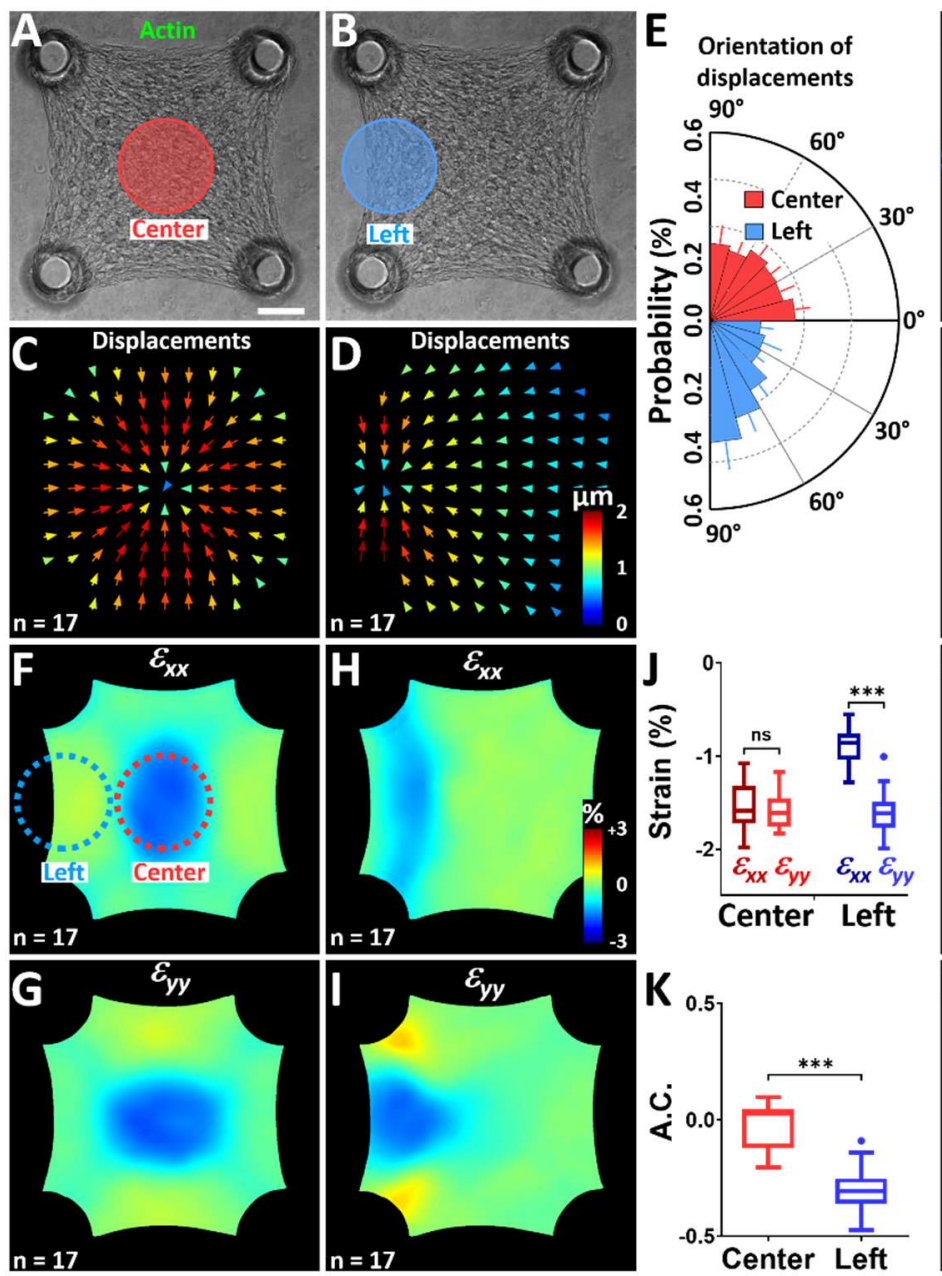
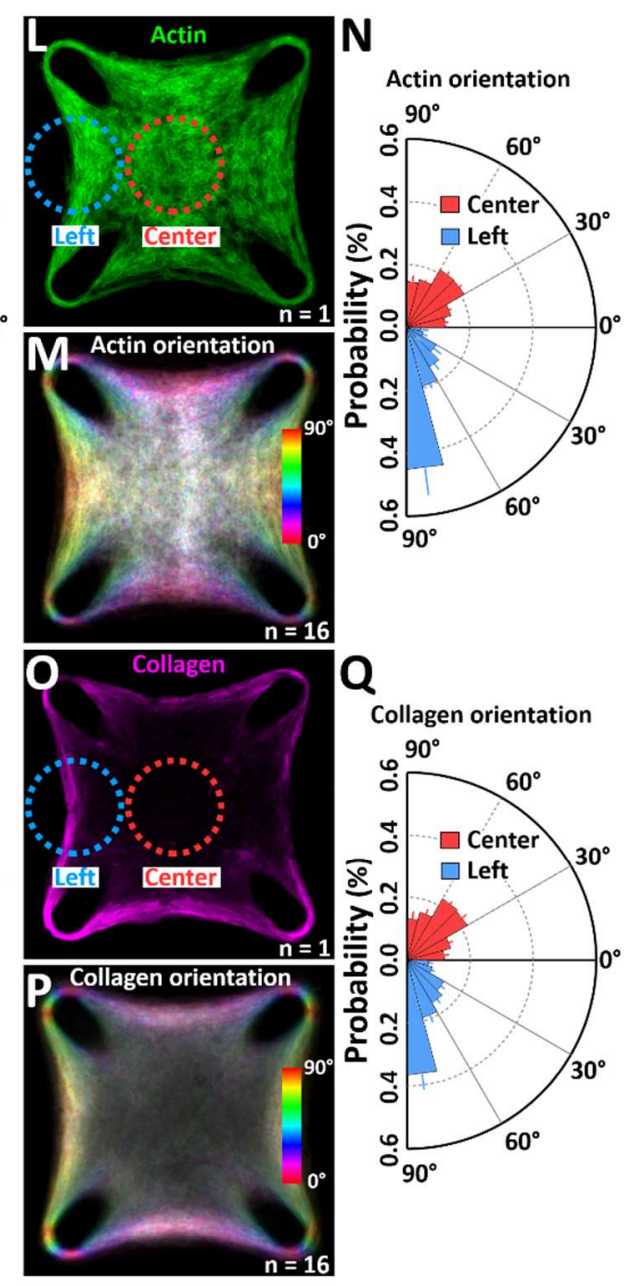

Figure 5. Optogenetically-mapped mechanical heterogeneities correlate with actin and collagen architecture. (A) Representative square microtissue where the red (A) and blue (B) discs represent the centered and eccentric $200 \mu \mathrm{m}$ diameter isotropic stimulation areas, respectively. Corresponding average displacement fields for the centered (C) and eccentric (D) stimulations. For readability reasons, only half of the vectors are represented. (E) Polar plot of the orientations of the corresponding displacement vectors. Data are presented as mean \pm SD with $n=17$ microtissues. Resulting average strain fields for centered $\left(\varepsilon_{x x}\right.$ in $F, \varepsilon_{y y}$ in $\left.\mathrm{G}\right)$ and eccentric $\left(\varepsilon_{x x}\right.$ in $\mathrm{G}, \varepsilon_{y y}$ in I) stimulations. Comparison of the average strain amplitudes $(\mathrm{J})$ and anisotropy coefficient A.C. (K) in the respective areas of stimulation. Data are presented as Tukey box plots with $\mathrm{n}=17$ microtissues. $* * * p<0.0001$. n.s. stands for nonsignificant (i.e. $p>0.05$ ). Confocal projection of the fluorescent staining of actin in a representative microtissue (L), average color-coded map (M) and polar plot (N) of the orientations of actin fibers in function of their localization, either in the center (red) or the left side (blue). Confocal projection of the fluorescent staining of collagen in a representative microtissue $(\mathrm{O})$, average color-coded map $(\mathrm{P})$ and polar plot $(\mathrm{Q})$ of the orientations of collagen fibers in function of their localization, either in the center (red) or the left side (blue). Data of actin and collagen orientations are presented as mean \pm SD with $n=16$ microtissues. Scale bar is $100 \mu \mathrm{m}$. 


\section{Discussion}

The mechanical properties of microtissues have been identified as major factors that regulate the physical integrity, contractility and function of such microtissues ${ }^{3-5,9}$. Yet, these properties have only been examined through the application of external loading and biochemical treatments ${ }^{6,11,26}$. By introducing optogenetic modulation of the cellular contractility to the microtissues, we were able to produce local, physiological, cell-generated mechanical perturbations while simultaneously tracking their spatial and temporal propagation. These perturbations were reversible and paralleled by a compression of the stimulated zone, while non-stimulated zones were stretched.

We thus used optogenetic cells as biological actuators to probe the mechanics of microtissues and demonstrate the viscoelastic behavior of fibroblasts/collagen microtissues. Indeed, strains were delayed in time when a local contraction was generated, but returned to their original state, over time, once the contraction was stopped. By tracking the maximum amplitudes of stress, compression and stretch in response to local light-activation of the contractility, we were able to plot hysteretic stress/strain curves typical of viscoelastic materials. Moreover, we demonstrated that the amplitude of stress and stretch were proportional to the size of the light-stimulation and so the number of stimulated cells, which allowed us to extract mechanical properties of microtissues from lightstimulations.

Using this approach, we found that boundary conditions affect both stiffness and viscosity of microtissues, whereas collagen density or integrity only impact tissue stiffness. In the long term, we showed that the tissue stiffness increases over maturation time, which correlates well with a celldriven cross-linking of the matrix during tissue maturation ${ }^{12,27}$. However, we did not measure any change of viscosity, suggesting that cellular intrinsic viscosity is independent on the maturation time. These results are in good agreement with previous work showing that, in the short term, the collagen matrix is the main determinant of the tissue stiffness ${ }^{9,11}$ while the actomyosin cytoskeleton is predominantly responsible for the viscoelasticity of microtissues ${ }^{13,28}$. 
Furthermore, we showed that the orientations and magnitudes of the light-induced contractions correlated with the collagen and actin orientation, highlighting the predominant role of fibrillar architecture in the spatial propagation of mechanical signals. Collagen organization has been shown to be key to the long-scale interactions between cells and their navigation through the $\mathrm{ECM}^{29-31}$. Here, the ability of our method to apply dynamic, internal mechanical perturbations, while simultaneously measuring the magnitude and spatio-temporal propagation of such perturbations opens an exciting avenue for examining the mechanical guidance of cell migration in fibrillar matrices. On a larger scale, our technique paves the way for mapping the mechanical heterogeneities in complex tissues, probing in real time the dynamic remodeling of tissue architecture or studying the impact of mechanical perturbations on tissue formation, maturation and function.

In conclusion, the combination of tissue engineering and optogenetics provides unique opportunities to quantitatively demonstrate the impact of physical and biological parameters on the generation, propagation and sensing of cell-generated mechanical perturbations in 3D tissues. Most importantly, our approach paves the way to probing the rheology of 3D tissues in real time and non-destructively, using their own constituting cells as internal actuators. These same attributes will likely provide valuable opportunities to elucidate how mechanical cues dynamically regulate tissue formation and function over space and time.

\section{Materials \& Methods}

\section{Cell culture, reagents and immuno-stainings}

Stable cell line NIH 3T3 fibroblasts with CIBN-GFP-CAAX and optoGEF-RhoA constructs $(<15$ passages, kindly provided by L. Valon and M. Coppey, Institute Curie, Paris, France) were cultured in Dulbecco's modified Eagle's medium (DMEM, Gibco Invitrogen) supplemented with $10 \%$ fetal bovine serum (FBS, Gibco Invitrogen), 100U/ml of penicillin and $100 \mu \mathrm{g} / \mathrm{ml}$ of streptomycin (Gibco Invitrogen), and kept at $37^{\circ} \mathrm{C}$ in an atmosphere saturated in humidity and containing 5\% CO2. Collagenase experiments were 
performed by incubating microtissues with $0.05 \mathrm{~g} / \mathrm{L}$ of collagenase type I (Sigma) for 10 minutes before thorough rinsing with PBS and replacement with growth medium.

Prior to immunostaining, samples were fixed in $4 \%$ paraformaldehyde (Sigma) and blocked with $2 \%$ BSA Sigma). Extracellular collagen was immuno-stained with a primary antibody against Collagen type I (Sigma) and detected with Alexa 647-conjugated, isotype-specific, anti-IgG antibodies (Invitrogen). Samples were then permeabilized with $0.5 \%$ Triton X-100 (Sigma) in TBS (50 mM Tris- $\mathrm{HCl}, 0.15 \mathrm{M} \mathrm{NaCl}$, pH 7.4) and actin was labeled with phalloidin-Atto488 (Sigma).

\section{Device fabrication and calibration}

SU-8 masters were fabricated following the technique described previously ${ }^{2,32}$. Briefly, layers of SU-8 photoresist (Microchem) were patterned onto silicon wafers by successive spin coat, alignment, exposure, and bake steps. Polydimethylsiloxane (PDMS, Sylgard 184, Dow-Corning) microfabricated tissue gauges ( $\mu$ TUGs) were molded from the SU-8 masters by double replication as described previously ${ }^{2,32}$. Different ratios of PDMS/curing agents were used to obtain PDMS with a stiffness ranging from 0.3 to $4 \mathrm{MPa}$, leading to cantilevers with a spring constant $\mathrm{k}$ ranging from $0.2 \mathrm{~N} / \mathrm{m}$ to 1.1 $\mathrm{N} / \mathrm{m}$, respectively.

Before cell seeding, the PDMS templates were sterilized in $70 \%$ ethanol followed by UV irradiation for 15 min and treated with $0.2 \%$ Pluronic F127 (Sigma) for 2 min to reduce cell adhesion. A reconstitution mixture, consisting of $1.5 \mathrm{mg} / \mathrm{mL}$ or $2.5 \mathrm{mg} / \mathrm{mL}$ liquid neutralized collagen I from rat tail (Advanced Biomatrix) was then added to the surface of the substrates on ice and templates were degassed under vacuum to remove bubbles in the liquid. A cooled suspension of 750,000 cells within reconstitution mixture was then added to the substrate and the entire assembly was centrifuged to drive the cells into the micropatterned wells, resulting in approximately 500 cells per well. Excess collagen and cells were removed by de-wetting the surface of the substrate before incubating at $37^{\circ} \mathrm{C}$ to induce collagen polymerization for $9 \mathrm{~min}$. Culture medium was then added to each substrate. Microtissues were kept in the incubator for $24 \mathrm{~h}$ before stimulation experiments, unless specified otherwise. 


\section{Optogenetic stimulation and microscopy}

Optogenetics stimulation and brightfield imaging were performed using an inverted Nikon Eclipse TI-2 microscope with an Orca flash 4.0 LT digital CMOS camera (Hamamatsu) and a CFI S Plan Fluor ELWD 20x/0.45 objective (Nikon). Light stimulations were achieved using a Mosaïc 3 digital micromirror device (DMD, Andor) and the $470 \mathrm{~nm}$ diode of a Spectra $\mathrm{X}$ light source (Lumencor) with a power of $1.74 \mathrm{~mW} / \mathrm{mm}^{2}$. The microtissues were stimulated once for $500 \mathrm{~ms}$, unless specified otherwise. The microscope was controlled using NIS Elements software (Nikon). Microtissues were maintained at 37 ${ }^{\circ} \mathrm{C}$ and $5 \% \mathrm{CO} 2$ in a top-stage incubator (Oko Lab).

Confocal images were obtained with a Leica laser scanning microscope (LSM SP8, Leica) equipped with a plan apochromatic 40x/1.30 objective (Leica). Cross-sectional areas were estimated from z-stack images while actin and collagen orientations were evaluated with the Orientation-J plug-in (http://bigwww.epfl.ch/demo/orientationj/) ${ }^{33}$ in Image J.

\section{Force measurement and strain measurement}

Cantilever spring constants were calculated utilizing a capacitive MEMS force sensor mounted on a micromanipulator as described previously ${ }^{4,34}$. The spring constant of the cantilevers were found to be $0.20 \pm 0.03 \mathrm{~N} / \mathrm{m}, 0.45 \pm 0.10 \mathrm{~N} / \mathrm{m}$ and $1.10 \pm 0.26 \mathrm{~N} / \mathrm{m}$ for PDMS/curing agent of 1:20, 1:10 and 1:4, respectively. For quantifying microtissue forces, brightfield images were taken every $30 \mathrm{~s}$ and the displacement of the top of the cantilevers was tracked using custom MATLAB script. Briefly, the displacement of the sharp contrast created by the edge of the cantilever heads was tracked over time by auto-correlation of the interpolated line profiles, allowing subpixel resolution. Tracking results were visually checked and faulty tracking discarded. Only tissues that were uniformly anchored to the tips of the cantilevers were included in the analysis.

The strain $\varepsilon$ in the microtissue was derived from the displacement field determined from the brightfield images using a particle image velocimetry (PIV) algorithm implemented as a Matlab toolbox (https://pivlab.blogspot.com/) ${ }^{35}$. Briefly, small sub images (interrogation areas) of an image pair 
consisting of the reference image at $t=0$ and the image of interest were cross-correlated in the frequency domain using FFT to derive the most probable particle displacement vector in the interrogation areas, with its $x$ and $y$ components $u$ and $v$, respectively. The size of the interrogation areas was successively reduced from $128 \times 128$ to $96 \times 96$ and finally $64 \times 64$ pixels (corresponding to $72 \times 72,54 \times 54$ and $36 \times 36 \mu \mathrm{m}$, respectively). The strain components were derived by numerical differentiation of $u$ and $v$ to both $x$ and $y$, comparing the displacement vector of each particle with the vectors of surrounding particles in a window of $3 \times 3$ particles ${ }^{36}$ :

$$
\varepsilon_{x x}=\frac{\partial u}{\partial x} ; \varepsilon_{y y}=\frac{\partial v}{\partial y} ; \varepsilon_{x y}=\frac{1}{2}\left(\frac{\partial u}{\partial y}+\frac{\partial v}{\partial x}\right)
$$

\section{Data analysis and statistics}

For each line graph, the mean represents the mean \pm standard deviation of $n$ microtissues $(n$ is defined in the caption of each figure). For each box plots, the box extends from the 25 th to 75 th percentiles, the median is plotted as a line inside the box and the whiskers extend to the most extreme data point that is no more than 1.5 times the interquartile range (IQR) from the edge of the box (Tukey style). Significances were assessed with Prism (GraphPad) by one-way analysis of variance, using Tukey's significant difference test $(p<0.001$ was considered significant).

\section{Aknowledgments}

A.M. acknowledges support from the University Grenoble Alpes Ph.D. fellowship program. G.C. acknowledges financial support from the ANR SupraWaves project, grant ANR-19-CE13-0028 of the French Agence Nationale de la Recherche (ANR). M.B. acknowledges financial support from the ANR MechanoSwitch project, grant ANR-17-CE30-0032-01. T.B. acknowledges fundings through CNRS grants (Actions Interdisciplinaires 2017, DEFI Instrumentation aux limites 2017, Tremplin@INP 2021, PEPS CNRS-INSIS 2021). This work was supported by the Center of Excellence of Multifunctional Architectured Materials "CEMAM" (n AN-10-LABX-44-01). 
The authors thank P. Moreau and I. Wang for their technical support, L. Vallon and M. Coppey for kindly providing the opto-RhoA fibroblasts, G. Chagnon and N. Briot for the mechanical characterization of the PDMS, as well as P. Recho for helpful discussions.

\section{References}

1. Vandenburgh, H. et al. Drug-screening platform based on the contractility of tissueengineered muscle. Muscle Nerve 37, 438-47 (2008).

2. Legant, W. R. et al. Microfabricated tissue gauges to measure and manipulate forces from 3D microtissues. Proc. Natl. Acad. Sci. USA 106, 10097-10102 (2009).

3. Sakar, M. S. et al. Cellular forces and matrix assembly coordinate fibrous tissue repair. Nat. Commun. 7, 11036 (2016).

4. Boudou, T. et al. A Microfabricated Platform to Measure and Manipulate the Mechanics of Engineered Cardiac Microtissues. Tissue Eng. Part A 18, 910-919 (2012).

5. West, A. R. et al. Development and characterization of a 3D multicell microtissue culture model of airway smooth muscle. Am. J. Physiol. Cell. Mol. Physiol. 304, L4-L16 (2013).

6. Asmani, M. et al. Fibrotic microtissue array to predict anti-fibrosis drug efficacy. Nat. Commun. 9, 1-12 (2018).

7. Vandenburgh, H. et al. Automated drug screening with contractile muscle tissue engineered from dystrophic myoblasts. FASEB J. 23, 3325-3334 (2009).

8. Hinson, J. T. et al. Titin mutations in iPS cells define sarcomere insufficiency as a cause of dilated cardiomyopathy. Science (80-. ). 349, 982-986 (2015).

9. Zhao, R., Chen, C. S. \& Reich, D. H. Force-driven evolution of mesoscale structure in engineered 3D microtissues and the modulation of tissue stiffening. Biomaterials 35, 50565064 (2014).

10. Walker, M., Rizzuto, P., Godin, M. \& Pelling, A. E. Structural and mechanical remodeling of the cytoskeleton maintains tensional homeostasis in 3D microtissues under acute dynamic stretch. Sci. Rep. 10, 7696 (2020).

11. Zhao, R., Boudou, T., Wang, W.-G., Chen, C. S. \& Reich, D. H. Decoupling Cell and Matrix Mechanics in Engineered Microtissues Using Magnetically Actuated Microcantilevers. Adv. Mater. 25, 1699-1705 (2013).

12. Zhao, R., Boudou, T., Wang, W.-G., Chen, C. S. \& Reich, D. H. Magnetic approaches to study collective three-dimensional cell mechanics in long-term cultures (invited). J. Appl. Phys. 115, 172616 (2014).

13. Walker, M., Godin, M., Harden, J. L. \& Pelling, A. E. Time dependent stress relaxation and recovery in mechanically strained 3D microtissues. APL Bioeng. 4, 036107 (2020).

14. Tischer, D. \& Weiner, O. D. Illuminating cell signalling with optogenetic tools. Nat. Rev. Mol. Cell Biol. 15, 551-558 (2014).

15. Oakes, P. W. et al. Optogenetic control of RhoA reveals zyxin-mediated elasticity of stress fibres. Nat. Commun. 8, 15817 (2017).

16. Valon, L., Marín-Llauradó, A., Wyatt, T., Charras, G. \& Trepat, X. Optogenetic control of cellular forces and mechanotransduction. Nat. Commun. 8, 14396 (2017).

17. Hall, A. Rho GTPases and the control of cell behaviour. Biochem. Soc. Trans. 33, 891-895 (2005).

18. Valon, L. et al. Predictive Spatiotemporal Manipulation of Signaling Perturbations Using Optogenetics. Biophys. J. 109, 1785-1797 (2015).

19. Eastwood, M., Mudera, V. C., McGrouther, D. A. \& Brown, R. A. Effect of precise mechanical 
loading on fibroblast populated collagen lattices: Morphological changes. Cell Motil. Cytoskeleton 40, 13-21 (1998).

20. Sander, E. A., Barocas, V. H. \& Tranquillo, R. T. Initial fiber alignment pattern alters extracellular matrix synthesis in fibroblast-populated fibrin gel cruciforms and correlates with predicted tension. Ann. Biomed. Eng. 39, 714-729 (2011).

21. Bose, P., Eyckmans, J., Nguyen, T. D., Chen, C. S. \& Reich, D. H. Effects of Geometry on the Mechanics and Alignment of Three-Dimensional Engineered Microtissues. ACS Biomater. Sci. Eng. 5, 3843-3855 (2019).

22. Trepat, X. et al. Universal physical responses to stretch in the living cell. Nature 447, 592-595 (2007).

23. Humphrey, D., Duggan, C., Saha, D., Smith, D. \& Käs, J. Active fluidization of polymer networks through molecular motors. Nature 416, 413-416 (2002).

24. Licup, A. J. et al. Stress controls the mechanics of collagen networks. Proc. Natl. Acad. Sci. 112, 9573-9578 (2015).

25. Legant, W. R., Chen, C. S. \& Vogel, V. Force-induced fibronectin assembly and matrix remodeling in a 3D microtissue model of tissue morphogenesis. Integr. Biol. 4, 1164 (2012).

26. Walker, M., Godin, M. \& Pelling, A. E. Mechanical stretch sustains myofibroblast phenotype and function in microtissues through latent TGF- $\beta 1$ activation. Integr. Biol. 12, 199-210 (2020).

27. Tomasek, J. J., Gabbiani, G., Hinz, B., Chaponnier, C. \& Brown, R. A. Myofibroblasts and mechano-regulation of connective tissue remodelling. Nat. Rev. Mol. Cell Biol. 3, 349-363 (2002).

28. Schierbaum, N., Rheinlaender, J. \& Schäffer, T. E. Combined atomic force microscopy (AFM) and traction force microscopy (TFM) reveals a correlation between viscoelastic material properties and contractile prestress of living cells. Soft Matter 15, 1721-1729 (2019).

29. Gjorevski, N., S. Piotrowski, A., Varner, V. D. \& Nelson, C. M. Dynamic tensile forces drive collective cell migration through three-dimensional extracellular matrices. Sci. Rep. 5, 11458 (2015).

30. Pakshir, P. et al. Dynamic fibroblast contractions attract remote macrophages in fibrillar collagen matrix. Nat. Commun. 10, 1850 (2019).

31. Ahmadzadeh, H. et al. Modeling the two-way feedback between contractility and matrix realignment reveals a nonlinear mode of cancer cell invasion. Proc. Natl. Acad. Sci. 114, E1617-E1626 (2017).

32. Ramade, A., Legant, W. R., Picart, C., Chen, C. S. \& Boudou, T. Microfabrication of a Platform to Measure and Manipulate the Mechanics of Engineered Microtissues. in Methods in Cell Biology (ed. Théry, M. P. and M.) 121, 191-211 (Elsevier Inc., 2014).

33. Rezakhaniha, R. et al. Experimental investigation of collagen waviness and orientation in the arterial adventitia using confocal laser scanning microscopy. Biomech. Model. Mechanobiol. 11, 461-473 (2012).

34. Klotzsch, E. et al. Fibronectin forms the most extensible biological fibers displaying switchable force-exposed cryptic binding sites. Proc. Natl. Acad. Sci. USA 106, 18267-18272 (2009).

35. Thielicke, W. \& Stamhuis, E. J. PIVlab - Towards User-friendly, Affordable and Accurate Digital Particle Image Velocimetry in MATLAB. J. Open Res. Softw. 2, (2014).

36. Stamhuis, E. J. \& Videler, J. J. Quantitative flow analysis around aquatic animals using laser sheet particle image velocimetry. J. Exp. Biol. 198, 283-294 (1995). 


\section{Supplemental Figures}

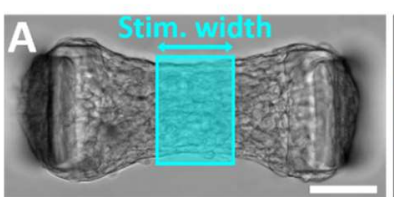

B

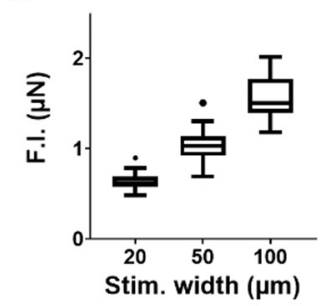

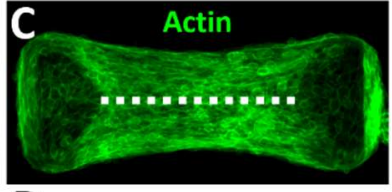

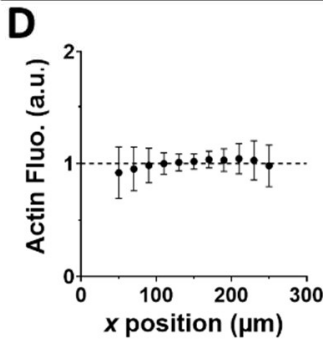

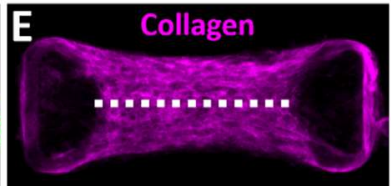

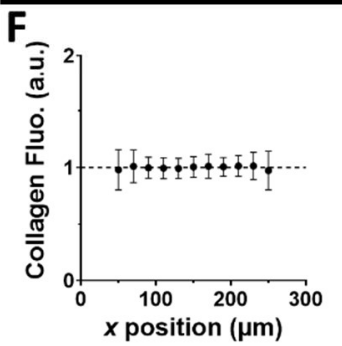

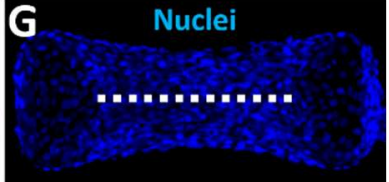

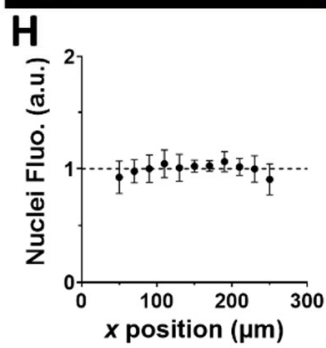

Supp. Figure 1. Light-induced contraction is proportional to the number of stimulated cells. Representative microtissue with the stimulation pattern in blue (A) and corresponding force increase (F.I.) in function of the stimulation width (B). Confocal projections and average fluorescence intensity along the dotted line for microtissues stained for actin $(C, D)$, collagen $(E, F)$ and nuclei $(G, H)$. Data are presented as mean $\pm S D$ with $n>15$ microtissues. Scale bar is $100 \mu \mathrm{m}$.

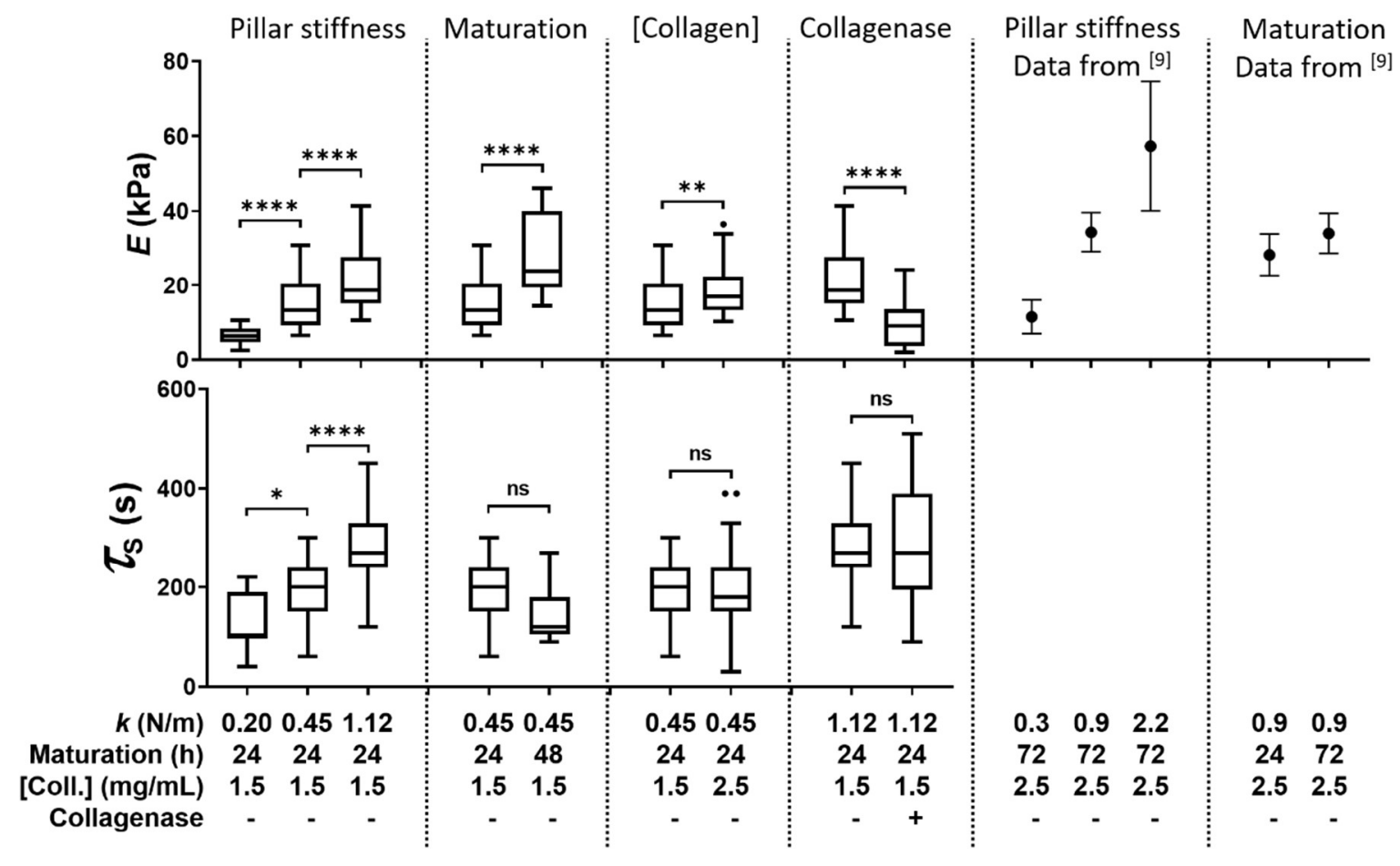

Supp. Figure 2. Optogenetic assessment of the rheological properties of microtissues. Elastic modulus $E$ and delay $\tau_{s}$ between maximum stress and stretch for three different spring constants $k$ of the micropillars, two durations of maturation, two collagen densities [Coll.] and the presence or absence of collagenase. Data are presented as Tukey box plots with $n>18$ microtissues. The two last columns present data from the literature ${ }^{9}$ for comparison. 


\section{Supplemental Movies}

Supp. Movie 1. Light-controlled local contraction of microtissues. Time lapse of a representative optomicrotissue whose left- then right-half are stimulated with blue light (represented by a blue rectangle) at $\mathrm{t}=40 \mathrm{~s}$ and $\mathrm{t}=21 \mathrm{~min}$, respectively. Upon blue light illumination, the microtissue contracts locally before slowly relaxing, as shown by the PIV-tracking of the displacements.

Supp. Movie 2. Anisotropic contraction of an opto-microtissue upon an isotropic stimulation. Temporal evolution of a representative opto-microtissue and the corresponding strain fields upon the stimulation in its center with a $50 \mu \mathrm{m}$ diameter discoidal light pattern (represented by a blue disc) at $\mathrm{t}$ $=1^{\prime} 20 "$. Despite an isotropic stimulation, the resulting strain is dominated by its $x$-axis component, as shown by the temporal evolution of $\varepsilon_{x x}$ and $\varepsilon_{y y}$.

Supp. Movie 3. Confocal reconstruction of an opto-microtissue. Z-stack (top) and 3D view (bottom) of a representative opto-microtissue stained for actin (in green), collagen (in magenta) and nuclei (in blue), highlighting the anisotropic orientation of actin and collagen fibers along the $x$-axis.

Supp. Movie 4. Light-induced local contractions evidence the viscoelastic properties of microtissues. Temporal evolution of a representative opto-microtissue, displacement field and $\varepsilon_{x x}$ strain field upon the stimulation of its left-half with blue light (represented by a blue rectangle) at $t=2 ' 30 "$. The stimulated half is strongly compressed, with a maximum at $t=7{ }^{\prime} 30 "$, while the non-stimulated half is stretched, with a maximum at $\mathrm{t}=99^{\prime} 30^{\prime \prime}$.

Supp. Movie 5. Contraction is proportional to the area of stimulation. Temporal evolution of a representative opto-microtissue, displacement field and $\varepsilon_{x x}$ strain field upon the illumination of its center by a $20 \mu \mathrm{m}$, a $50 \mu \mathrm{m}$ and a $100 \mu \mathrm{m}$ wide stimulation (represented by blue rectangles) at $\mathrm{t}=2$ $\min , t=22 \min$ and $t=42 \min$, respectively.

Supp. Movie 6. Isotropic contraction of the center of an opto-microtissue. Temporal evolution of a representative opto-microtissue, displacement field and strain fields upon the stimulation in its center with a $200 \mu \mathrm{m}$ diameter discoidal light pattern (represented by a blue disc) at $\mathrm{t}=40 \mathrm{~s}$. The resulting contraction is isotropic, as shown by the displacement field and the similar amplitudes of $\varepsilon_{x x}$ and $\varepsilon_{y y}$.

Supp. Movie 7. Anisotropic contraction of the side of an opto-microtissue. Temporal evolution of a representative opto-microtissue, displacement field and strain fields upon the stimulation on its left side with a $200 \mu \mathrm{m}$ diameter discoidal light pattern (represented by a blue disc) at $\mathrm{t}=40 \mathrm{~s}$. The resulting contraction is anisotropic, as shown by the displacement field and the differences in amplitude between $\varepsilon_{x x}$ and $\varepsilon_{y y}$.

Supp. Movie 8. Confocal reconstruction of an opto-microtissue. Z-stack (left) and 3D view (right) of a representative opto-microtissue stained for actin (in green) and collagen (in magenta), highlighting the anisotropic orientation of actin and collagen fibers along the sides, while the center is mostly disorganized. 